\title{
Towards Efficient, Scalable and Reliable Network Functions Virtualization in Distributed Cloud Environments
}

\author{
Brigitte Jaumard \\ Concordia University, \\ 7141 Rue Sherbrooke O, Montréal, QC H4B 1R6, Camada
}

The recent emergence of concepts like Cloud Computing, Software Defined Networking (SDN), and ultimately, Network Functions Virtualization (NFV) offers new promises to Telecommunication Service Providers (TSPs): more flexibility to further open up their network capabilities and services to users and other services, and the ability to deploy or support new network services faster and cheaper so as to realize better service agility.

Indeed, Network Function Virtualization (NFV) aims to abstract the functionality of traditional proprietary hardware into software as Virtual Network Functions (VNFs), which can run on commercial off-the-shelf (COTS) servers. It leads to new network service provisioning best practices by decoupling software from hardware, deploying flexible network functions and managing a better scaling with respect to dynamic traffic. Current standardization effort looks for an NFV orchestration that is handle network services that each consists of a set of VNFs interconnected by virtual links, which is referred to as a service function chain (SFC).

We will discuss the best strategies in order to design efficient, scalable and reliable NFV management and orchestration in the context of a distributed cloud environment. 